\title{
4th order tensors for multi-fiber resolution and segmentation in white matter
}

\author{
Temesgen Bihonegn* \\ Avinash Bansal \\ Jan Slovák \\ temesgent275@gmail.com \\ avinashbansal26@gmail.com \\ slovak@muni.cz \\ Faculty of Science, Masaryk University \\ Brno, Czech Republic
}

\author{
Sumit Kaushik \\ kaushsum@cmp.felk.cvut.cz \\ Faculty of Electrical Engineering \\ Czech Technical University \\ Prague, Czech Republic
}

\begin{abstract}
Since its inception, DTI modality has become an essential tool in the clinical scenario. In principle, it is rooted in the emergence of symmetric positive definite (SPD) second-order tensors modelling the difusion. The inability of DTI to model regions of white matter with fibers crossing/merging leads to the emergence of higher order tensors. In this work, we compare various approaches how to use 4th order tensors to model such regions. There are three different projections of these 3D 4th order tensors to the 2nd order tensors of dimensions either three or six. Two of these projections are consistent in terms of preserving mean diffusivity and isometry. The images of all three projections are SPD, so they belong to a Riemannian symmetric space. Following previous work of the authors, we use the standard k-means segmentation method after dimension reduction with affinity matrix based on reasonable similarity measures, with the goal to compare the various projections to 2nd order tensors. We are using the natural affine and log-Euclidean (LogE) metrics. The segmentation of curved structures and fiber crossing regions is performed under the presence of several levels of Rician noise. The experiments provide evidence that 3D 2nd order reduction works much better than the $6 \mathrm{D}$ one, while diagonal components (DC) projections are able to reveal the maximum diffusion direction.
\end{abstract}

\section{CCS CONCEPTS}

- Mathematics of computing $\rightarrow$ Mathematical analysis; $\bullet$ Computing methodologies $\rightarrow$ Modeling and simulation; $\bullet$ Applied computing $\rightarrow$ Bioinformatics.

\section{KEYWORDS}

Diffusion Tensor Imaging, Tensor Reduction, Segmentation, Diagonal component (DC), Fiber Resolution

Permission to make digital or hard copies of all or part of this work for personal or classroom use is granted without fee provided that copies are not made or distributed for profit or commercial advantage and that copies bear this notice and the full citation on the first page. Copyrights for components of this work owned by others than ACM must be honored. Abstracting with credit is permitted. To copy otherwise, or republish, to post on servers or to redistribute to lists, requires prior specific permission and/or a fee. Request permissions from permissions@acm.org.

ICBBE '20, November 6-9, 2020, Kyoto, Japan

(C) 2020 Association for Computing Machinery.

ACM ISBN 978-1-4503-8822-1/20/11 ..\$15.00

https://doi.org/10.1145/3444884.3444892

\section{ACM Reference Format:}

Temesgen Bihonegn, Avinash Bansal, Jan Slovák, and Sumit Kaushik. 2020. 4 th order tensors for multi-fiber resolution and segmentation in white matter. In 2020 7th International Conference on Biomedical and Bioinformatics Engineering (ICBBE '20), November 6-9, 2020, Kyoto, Japan. ACM, New York, NY, USA, 7 pages. https://doi.org/10.1145/3444884.3444892

\section{INTRODUCTION}

Based upon NMR principles Lauterbur, [23], developed MRI technique to render 3D images. Diffusion-weighted images have come a long way since then. Diffusion Tensor Imaging (DTI) was introduced to model diffusion in biological tissues in [5] and [17]. It proved phenomenal in clinical studies to probe into cerebral white matter structures. It enabled us to infer the microstructures of tissues in-vivo and noninvasively. The introduction of more diffusion gradient directions revealed more detailed structures (in DTI the number of independent parameters for each voxel is six). This acquisition protocol is known as high angular diffusion imaging (HARDI). Since then various modalities based upon HARDI have been proposed [24][38]. The monoexponential Stejskal-Tanner equation is assumed to model the D-MRI (Diffusion-Magnetic Resonance Imaging) principle. The DTI model is restricted to produce second-order tensors. These tensors are effective in modeling the regions where fibers are not crossing, merging or touching. Various works have utilized this space for processing these tensors [13][14][16][22][25][32]. Another common approach is to use qspace like DSI and Q-Ball. Diffusion is a physical process, methods in [2][3] ensure the full symmetry and positive definiteness of higher order tensors. Every symmetric tensor can be represented as a homogeneous polynomial [3]. This helps in finding the maxima of the Apparant Diffusion Coefficient (ADC) profiles. One reason to use higher order tensors is that they encode diffusion geometry without the need of evaluating spherical harmonics from the diffusion profiles. Another advantage comes from the observation that the computation of coefficients of lower-order tensors can be obtained from coefficients of higher-order tensor using linear relations [28][30] without refitting of DMR signal. The works in [26][39] suggest that in intersection regions these tensors fail to orient properly with the underlying direction of actual fibers. The issue of reorientation is resolved in work [39] and the approach is referred to as Cartesian Tensor-fiber orientation distribution (CT-FOD). 
In [9][10], the authors approached the segmentation problem by considering the individual fiber bundles to lie in $1 \mathrm{D} / 2 \mathrm{D} / 3 \mathrm{D}$ subspaces, depending upon the numbers of the intersecting fibers. In [18], 5D non-linear geometry is employed to segregate fiber tracts. This approach proved advantageous over the 3D Euclidean space assumption. The surface evolution [33] in Riemannian space can segment such curved structures. Works [25][35] used the Hilbert sphere in infinite dimension and mapped the data to lower dimension for segmentation.

In this work, we discuss the order reduction of the 3D 4th order tensors. This reduction yields 2 nd order tensors in $3 \mathrm{D}$ and $6 \mathrm{D}$. Further, these reductions are SPDs and therefore lie in Riemannian symmetric space. These SPD data belong to a log-normal distribution. We computed the variance of the data using this property. Subsequently, a non-linear dimensionality reduction method known as Laplacian-Eigenmap clustering is utilized for the extraction of anisotropic regions in both synthetic and real images. The results infer that systematic order reduction of tensors is useful and it is robust under noise. We segmented with single and two crossing fibers with various complex configurations. Another observation is that the diagonal component projection obtained from flattened 3D 4th order tensor can reveal the direction of maximum diffusion. Our experiments are discussed in detail in section 5 .

\section{BACKGROUND}

\subsection{Diffusion Modelling}

The Stejskal-Tanner equation represents a mono-exponential model of water molecules diffusing in tissues given by:

$$
\begin{gathered}
S(b, v)=S_{0} \exp (-b D(v)), \\
D(v)=\sum_{1}^{n} \sum_{j_{1}=1}^{3} \sum_{j_{2}=1}^{3} \sum_{j_{3}=1}^{3} \ldots \sum_{j_{n}=1}^{3} D_{j_{1} j_{2} j_{3} \ldots j_{n}} v_{j_{1}} v_{j_{2}} v_{j_{3}} \ldots v_{j_{n}},
\end{gathered}
$$

where $v_{\ell}$ is $\ell$ th magnetic gradient component and $\|v\|=1 . S(v)$ is the attenuated signal when gradient pulse is applied and $b$ is diffusion weighting coefficient. Due to antipodal symmetry and physicality of diffusion process, the higher order tensors from the above equation are positive definite and of even order. For the same reasons, these tensors are fully symmetric. Due to the full symmetry, the number of independent coefficients for kth order tensor is reduced from $3^{k}$ to $\frac{1}{2}(k+1)(k+2)$. The seminal work of Tuch et. al. [36] is based upon the conjuncture that increasing number of gradient directions should be able to reveal geometry of the biological tissues. For 4 th order tensors, 81 coefficients reduce to 15 .

\subsection{Linear Algebra}

Let $\mathrm{V}$ be $n$-dimensional vector space defined over real numbers $\mathbb{R}$. 4 th order tensors form a vector space of dimension $n^{4}$, where $n=3$ in our case. We are interested in the so called Cartesian tensors, i.e., the coordinate description of the tensor in a fixed orthonormal basis of the vector space V. Thus, a second order tensor can be viewed as 2-dimensional array of scalars. Under orthogonal basis, tensors can be also represented as $k$-linear forms

$$
T\left(X_{1}, \ldots, X_{k}\right)=\sum_{j=1}^{n} T_{j_{1} \ldots j_{k}} x_{1}^{j_{1}} \ldots x_{k}^{j_{k}}
$$

where the tensor $T$ is evaluated at vectors $X_{i}$. Using an orthonormal basis $e^{i}$, for $i=1,2 \ldots, n$, a 4 th order tensor $T$ is written as

$$
T^{(4)}=\sum_{1 \leq i, j, k, l}^{n} T_{i j k l}^{(4)} e_{i} \otimes e_{j} \otimes e_{k} \otimes e_{l},
$$

where the individual terms $e_{i} \otimes e_{j} \otimes e_{k} \otimes e_{l}$ form the induced orthonormal basis of the space of fourth order tensors. Thus, there is the induced scalar product of two tensor $S=S_{i_{1} \ldots i_{k}}, R=R_{i_{1} \ldots i_{k}}$, cf. [19], the so called dot product

$$
S \bullet R=\sum_{i_{1}, \ldots, i_{k}=1}^{n} S_{i_{1} \ldots i_{k}} R^{i_{i} \ldots i_{k}} .
$$

For $k=2$, the scalar product of two tensors becomes

$$
S \bullet R=\sum_{i, j=1}^{n} S_{j}^{i} R_{i}^{j}=\operatorname{trace}\left(R^{T} S\right) .
$$

The corresponding Euclidean distance measure on the space of tensors becomes

$$
d_{E}(S, R)=\|S-R\| .
$$

Exploiting the inner product, the 4th order tensors can be identified as mappings between second order tensors. This means, we can represent $T^{(4)}$ using 2 nd order tensor components. For $1 \leq i, j, k, l \leq n$ we arrive at

$$
T^{(4)}=\sum_{i j k l} T_{i j k l}^{(4)} e_{i} \otimes e_{j} \otimes e_{k} \otimes e_{l}=\sum_{I J} T_{I J}^{(2)} e_{I} \otimes e_{J}
$$

where $e_{I(i, j)}=e_{i} \otimes e_{j}, e_{J(k, l)}=e_{k} \otimes e_{l}$. In view of this identification, they exhibit three types of symmetries:

(1) Major Symmetry: $T_{i j k l}=T_{k l i j}, 1 \leq i, j, k, l \leq n$, which corresponds to symmetric mappings between the second order tensors.

(2) Minor Symmetry: $T_{i j k l}=T_{j i k l}=T_{i j l k}$, which corresponds to the restriction to symmetric second order tensors, with symmetric values.

(3) Total Symmetry: $T_{i j k l}=T_{\sigma(i) \sigma(j) \sigma(k) \sigma(l)}$, for every permutation $\sigma$, which means both of the previous symmetries together.

In diffusion process, the even order tensors obey total symmetry. These 3D 4th order tensors can be represented as homogeneous polynomials (say in coordinates $x, y, z$ ) of degree 4 , built of monomials $p_{i j k} x^{i} y^{j} z^{k}$ with $i+j+k=4$. The relation between the coefficient of the polynomial and that of tensor is represented by the equation: $\frac{i ! j ! k !}{4 !} p_{i j k}=D_{j_{1} j_{2} j_{3} j_{4}}$, where $j_{\ell}$ are one of the terms $x, y, z$. Vector representations of 4 th order and 2nd order tensors are unable to reveal their geometric properties, like the distribution of eigenvalues and eigenvectors of the tensorial form [6]. By loosing the tensorial form, it is not possible to see the effect of rotation of the coordinate system on the distribution of tensor [31], etc. For these reasons, we are interested in the reductions which are obtained systematically while preserving important information. 


\section{MAPPINGS}

\subsection{D 2nd order representation}

The appearance of 4th order tensor is known in various fields [7][40]. In material science, they are known to classify materials based upon their elasticity [37][8][12]. They model the material symmetries, which is reflected in the invariance of components of the tensor with permutation of indices. A 3D 4th order tensor with minor symmetry can be written as Voigt contracted notation:

$$
D=\left(\begin{array}{cccccc}
D_{x x x x} & D_{x x y y} & D_{x x z z} & \sqrt{2} D_{x x y z} & \sqrt{2} D_{x x x z} & \sqrt{2} D_{x x x y} \\
D_{y y x x} & D_{y y y y} & D_{y y z z} & \sqrt{2} D_{y y y z} & \sqrt{2} D_{y y x z} & \sqrt{2} D_{y y x y} \\
D_{z z x x} & D_{z z y y} & D_{z z z z} & \sqrt{2} D_{z z y z} & \sqrt{2} D_{z x x z} & \sqrt{2} D_{z z x y} \\
\sqrt{2} D_{y x x} & \sqrt{2} D_{y z y y} & \sqrt{2} D_{y z z z} & D_{y z y z} & D_{y z x z} & D_{y z x y} \\
\sqrt{2} D_{x x x} & \sqrt{2} D_{x z y y} & \sqrt{2} D_{x z z z} & D_{x z y z} & D_{x z x z} & D_{x z x y} \\
\sqrt{2} D_{x y x x} & \sqrt{2} D_{x y y y} & \sqrt{2} D_{x y z z} & D_{x x y z} & D_{x y x z} & D_{x y x y}
\end{array}\right)
$$

With the following extra equalities the tensor exhibits the total symmetry.

$$
\begin{aligned}
& D_{x x y y}=D_{x y x y}, D_{x x z z}=D_{x z x z}, D_{y y z z}=D_{y z y z} \\
& D_{x x y z}=D_{x y x z}, D_{y y x z}=D_{x y y z}, D_{z z x y}=D_{x z y z}
\end{aligned}
$$

In this isometric notation, it is a $6 \mathrm{D}$ second order tensor. This tensor is an SPD and so, lies in Riemannian symmetric space. Their positive definiteness is a favourable property to justify diffusion as a physical phenomenon. The conversion between 3D 4th order tensor coefficient and 6D 2nd order is obtained through equation (8). The factor 2 and $\sqrt{2}$ ensures isomorphism between the two spaces [27][34].

\section{$3.23 \mathrm{D} 2 \mathrm{nd}$ order reduction}

There are many ways to represent the 4 th order tensor. An option preserving the metric is obtained via spherical harmonics and the corresponding linear mapping is given by the formulae in [28]:

$$
\begin{aligned}
D_{x x} & =\frac{3}{35}\left(9 D_{x x x x}+8 D_{x x y y}+8 D_{x x z z}-D_{y y y y}-D_{z z z z}-2 D_{y y z z}\right) \\
D_{y y} & =\frac{3}{35}\left(9 D_{y y y y}+8 D_{x x y y}+8 D_{y y z z}-D_{x x x x}-D_{z z z z}-2 D_{x x z z}\right) \\
D_{x x} & =\frac{3}{35}\left(9 D_{z z z z}+8 D_{x x z z}+8 D_{y y z z}-D_{x x x x}-D_{y y y y}-2 D_{x x y y}\right) \\
D_{x y} & =\frac{6}{7}\left(D_{x x x y}+D_{y y y x}+D_{z z x y}\right) \\
D_{x z} & =\frac{6}{7}\left(D_{x x x z}+D_{z z z x}+D_{y y x z}\right) \\
D_{y z} & =\frac{6}{7}\left(D_{y y y z}+D_{z z z y}+D_{x x y z}\right)
\end{aligned}
$$

The formulation of this reduction given by equation (9) is consistent as mean diffusivity is proportional as follows:

$$
\operatorname{trace}\left(T^{(2)}\right)=\frac{3}{5} \operatorname{trace}\left(T^{(4)}\right)
$$

The reader is referred to [28] for details.

\subsection{Flattening of 4th order tensor}

Another approach to describe 4th order tensors is by unfolding the tensor, arranging the tensor as a matrix. Thus, a general $r$ th order tensor $T^{(r)}$ can be expressed as a matrix of $(r-2)$ nd order tensors:

$$
T^{(r)}=\left(\begin{array}{lll}
T_{x x}^{r-2)} & T_{x y}^{(r-2)} & T_{x z}^{(r-2)} \\
T_{y x}^{(r-2)} & T_{y y}^{(r-2)} & T_{y z}^{(r-2)} \\
T_{z x}^{(r-2)} & T_{z y}^{(r-2)} & T_{z z}^{(r-2)}
\end{array}\right)
$$

We deal with $r=4$, but our discussion is extendable to higher orders. The diagonal components of this representation are SPD [20]. Thus, we obtain 3 2nd order SPD out of one 4 th order tensor. Choosing the coordinates to diagonalize one of them leaves 15 free parameters, exactly as for the 4th order tensors. This method is called the diagonal component (DC) projection.

\section{RIEMANNIAN MANIFOLD CLUSTERING}

For processing the fields of 3D and 6D 2nd order tensors, we use the so called affine and Log-Euclidean (LogE) metrics [19]. Exponential map is a function that maps each symmetric matrix to an SPD. The inverse of the exponential map is the logarithmic map. We may use these inverse mapping at each fixed SPD matrix $p$. Several authors discussed various metrics suitable for statistics explored in imaging, see [19] for a survey, including the spectral similarity measures. The affine invariant metric is the natural metric of the Riemannian symmetric space, but it is computationally slow with involvements of inverse, square root and logarithmic operations. Moreover, this metric has limitation like swelling effect. The geodesic distance between two SPD tensors $p, x$ is computed as

$$
d_{A}(p, x)=\left\|\log _{p}(x)\right\| \text {. }
$$

In the ambient Euclidean space, the SPD matrices lie in the interior of a convex cone and the affine metric turns it into a complete Riemannian manifold. See [15] for more information.

The LogE metric is due to [1]. This metric is based upon the observation that matrix exponential of symmetric matrices is diffeomorphic to the space of SPDs. For two SPD matrices $p_{1}, p_{2}$,

$$
d_{\operatorname{LogE}}\left(p_{1}, p_{2}\right)=\left\|\log \left(p_{1}\right)-\log \left(p_{2}\right)\right\| .
$$

The studies [1][11][19] also indicate that LogE metric is better in preserving anisotropy measure. The white matter is modelled as a tensor with an anisotropy. This measure is crucial in evaluation of statistics of tensors, white matter tractography and segmentation. The spectral similarity measures perform even better, but we are using the affine and LogE metrics to compare the projections here.

We use the Lapacian Eigen Map (LE) for projecting the non linear data to lower dimension. For this projection affinity matrix is computed as:

$$
w_{i j}=\exp \left(-\frac{\operatorname{dist}\left(p_{i}, p_{j}\right)^{2}}{\sigma^{2}}-\frac{\|i-j\|^{2}}{w_{e}}\right)
$$

The first term evaluates affinity between data in Riemannian space whereas the 2nd term provides similarity in the image space (with $\|i-j\|$ being a suitably blown-up Euclidean distance ensuring robustness with respect to noise). These terms ensure extraction of the fiber structure from the background. The variance is evaluated respecting the log-normal distribution of the SPD diffusion tensor data [20]. The coefficient $w_{e}$ is experimentally chosen and depends upon the size of window.

\section{EXPERIMENT AND RESULTS}

We simulated synthetic images (64 Gradient direction with $b=1500$ $\mathrm{s} / \mathrm{mm}^{2}$ ) using adaptive kernel method [4]. The Fig. 1 (a)-(d) shows 4 th order tensor ODF where angle difference between the two fibers are $30^{\circ}, 45^{\circ}, 75^{\circ}$ and $90^{\circ}$. The maxima of these ODFs do not necessarily align with actual underlying fibers. Another issue with these ODFs is fuzziness in the maxima. In Fig.1 (a) and (b) the pointing circle in red indicates the maxima at angles in between the range. These maxima are at wrong position, right positions are indicated by the blue circle. 
Table 1: Execution time for DC vs. CT-FOD methods

\begin{tabular}{|c|c|c|c|c|c|c|c|c|c|c|c|c|c|c|}
\hline \multicolumn{15}{|c|}{ Comparison Table } \\
\hline \begin{tabular}{|l} 
Angle \\
between \\
fibers
\end{tabular} & $\begin{array}{l}\text { Difference } \\
\text { underlying }\end{array}$ & 00 & 10 & 20 & 30 & 40 & 50 & 60 & 70 & 80 & 90 & 100 & 110 & 120 \\
\hline \multirow[b]{2}{*}{ Time in Sec } & DC Method & 0.0014 & $|0.0013|$ & 0.0011 & $\mid 0.0012$ & 0.0095 & $|0.0001|$ & $|0.0001|$ & 0.0001 & 0.0002 & $|0.0001|$ & 0.0001 & 0.0001 & 0.0001 \\
\hline & CT ODFs & 3.1437 & $|1.7980|$ & 2.0849 & 1.6177 & 1.6555 & $\mid 2.0732$ & $\mid 2.0209$ & 2.0216 & 2.1774 & $|1.6799|$ & 2.044 & 2.1078 & 2.1189 \\
\hline
\end{tabular}

Table 2: Segmentation of the fiber and background (Dice coefficient)

\begin{tabular}{|c|c|c|c|c|c|c|c|c|c|c|c|}
\hline & & \multicolumn{5}{|c|}{ 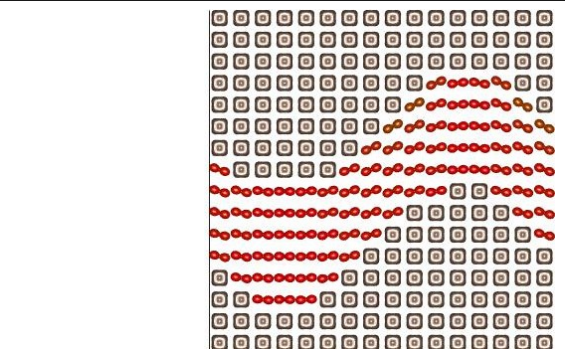 } & & & & & \\
\hline \multirow{2}{*}{ Metric } & \multirow{2}{*}{$\begin{array}{l}\text { Method/ Rician } \\
\text { Noise Level }\end{array}$} & \multicolumn{5}{|c|}{ Back Ground } & \multicolumn{5}{|c|}{ Fiber 1} \\
\hline & & 0.00 & 0.02 & 0.04 & 0.06 & 0.08 & 0.00 & 0.02 & 0.04 & 0.06 & 0.08 \\
\hline \multirow[b]{2}{*}{ Affine } & 3D Mapping & 1.000 & 0.994 & 0.995 & 0.998 & 0.998 & 1.000 & 0.970 & 0.973 & 0.995 & 0.995 \\
\hline & 6D Mapping & 0.937 & 0.984 & 0.950 & 0.985 & 0.953 & 0.874 & 0.937 & 0.862 & 0.940 & 0.860 \\
\hline \multirow[b]{2}{*}{$\log E$} & 3D Mapping & 0.967 & 0.983 & 0.975 & 0.996 & 0.984 & 0.906 & 0.939 & 0.909 & 0.955 & 0.911 \\
\hline & 6D Mapping & 0.948 & 0.982 & 0.967 & 0.956 & 0.949 & 0.852 & 0.937 & 0.844 & 0.911 & 0.875 \\
\hline
\end{tabular}

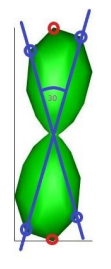

(a) $30^{\circ}$

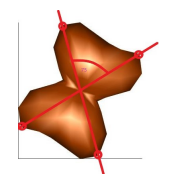

(c) $75^{\circ}$

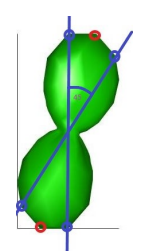

(b) $45^{\circ}$



(d) $90^{\circ}$
Figure 1: 4th order ODF with various angle differences between the two fibers

Fig. 3 displays fiber orientation error in the presence of Rician noise at various angle differences between the two fibers. The vertical bar shows standard deviation for 50 repeated experiments at each level of noise. The principal eigenvectors of the diagonal components (DC) can discern directions of maximum diffusion. The CT-FOD

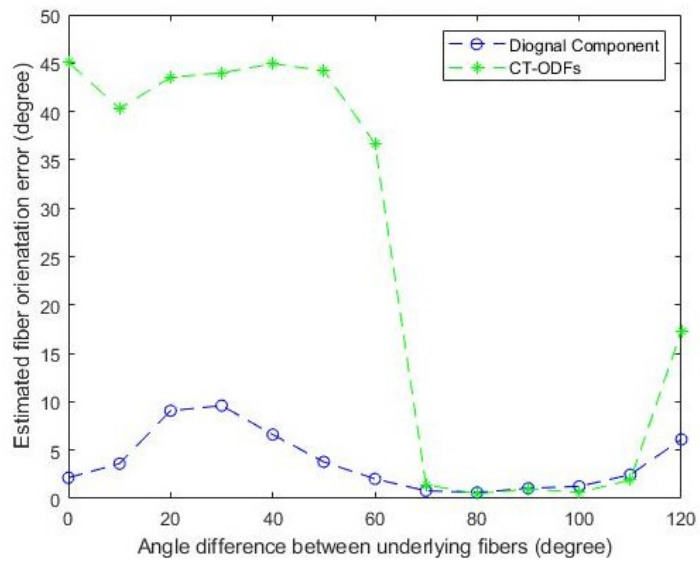

(a)

Figure 2: Comparison of DC vs. CT-FOD

method is based on the signal deconvolution [39]. This approach uses a subroutine to find maxima of the reoriented ODFs. We compared evolution of such maxima with our method. These maximas are directions of prominent diffusion directions. We generated ODFs 


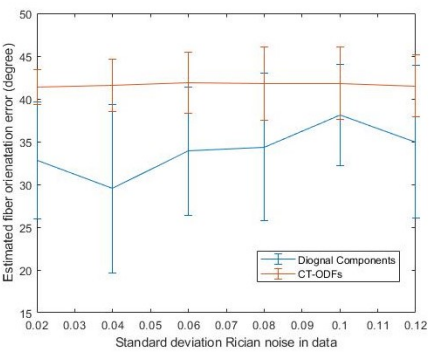

(a) $30^{\circ}$

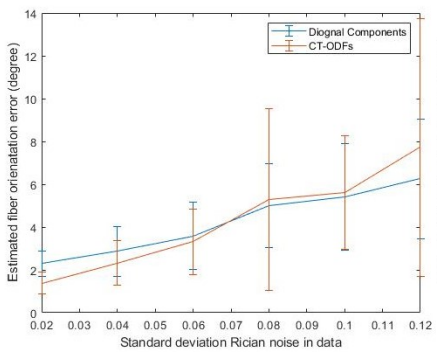

(c) $75^{\circ}$

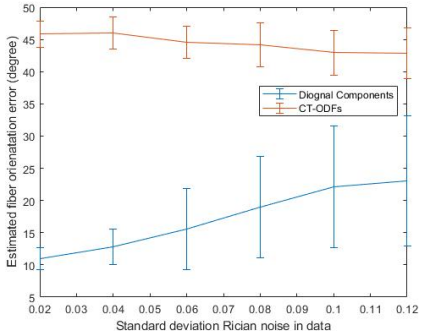

(b) $45^{\circ}$.



(d) $90^{\circ}$
Figure 3: Standard deviation of orientation error in the presence of Rician noise

for two fibers crossing at known angles and estimated fiber orientation error due to the DC and CT-FOD approach. The comparison is shown in Fig. 2. The performance of both the methods is similar within angle-difference range $70^{\circ}-110^{\circ}$ but for angle-differences outside this range the DC method performs better than CT-FOD. In all cases, the DC method shows lower orientation errors. As we approach within the above range their performances converge.

Table 1 displays the relative execution time. This experiment is conducted on machine with 16 GB RAM and Processor Intel(R) Core(TM) i5-7500 CPU @ 2.70GHz 2.71GHz. The DC method is about $10^{3}$ times faster. The independent 15 coefficients are arranged at fixed positions, thus computation of the three diagonal components is straightforward. Consequently, it jumps the optimization step which needs to find the maxima of ODF in CT-FOD method.

Table 2 shows average segmentation results in terms of Dice coefficients under various levels of Rician noise [29]. We created a data bank of 30 synthetic configurations having one (curved/linear) fiber. The performance of 3D 2nd order mapping with affine metric is slightly better than all other combinations. We performed similar tests including crossing fibers with different complexities. The comparison of the projections and metrics results in the same conclusion. We have also performed similar tests on real images. See Fig. 5, 6 for one example. Again, the 3D 2nd order affine metric choice outperforms the others.

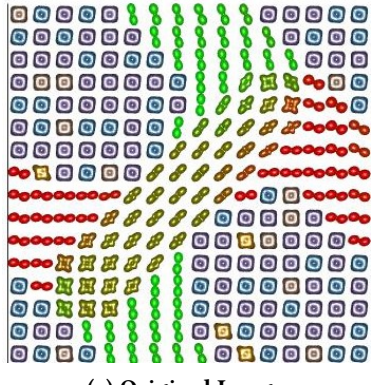

(a) Original Image

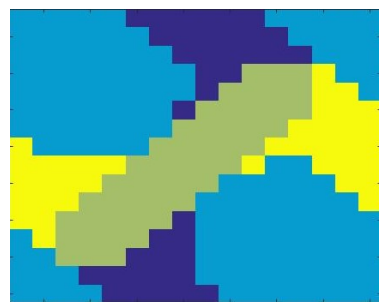

(b) 3D, Affine

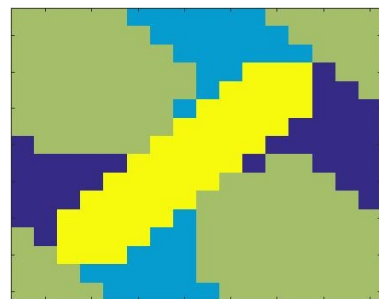

(d) 6D, Affine

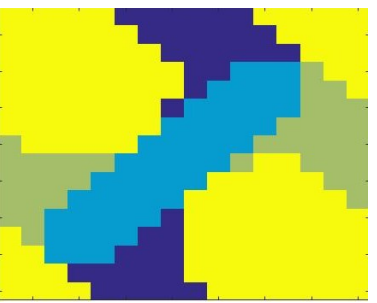

(c) $3 \mathrm{D}, \log \mathrm{E}$



(e) $6 \mathrm{D}, \log \mathrm{E}$
Figure 4: Four regions segmentation via the mappings (3D and 6D 2nd order tensors) under Affine and LogE metrics

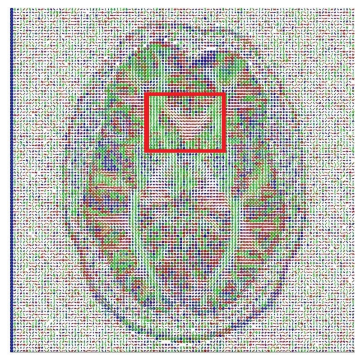

(a) Tensor Field Real Image

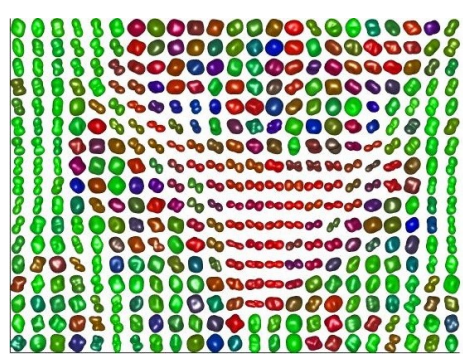

(b) Rectangular section shown in (a)
Figure 5: Real Image

\section{CONCLUSION}

The experiments have shown that the $6 \mathrm{D}$ projection of 4 th order tensors is more sensitive to noise than the $3 \mathrm{D}$ projection. Previous work of the authors [20] showed that the 3D DC projections, together with the spectral metrics perform, even better. 


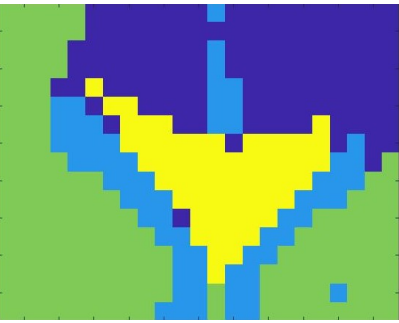

(a) 3D, Affine

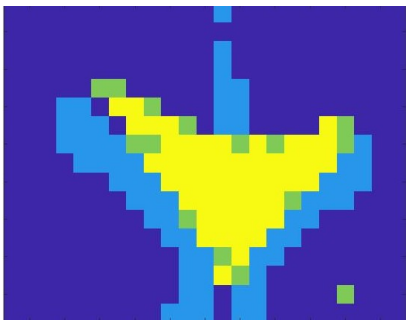

(b) 6D, Affine



(c) $3 \mathrm{D}, \log \mathrm{E}$



(d) $6 \mathrm{D}, \log \mathrm{E}$

Figure 6: Segmentation result

In segmentation application, [21] crossing regions are considered as a unit, therefore, the orientation of individual fibers has no effect on the outcome. The diagonal components of the flattened 4 th order tensor effectively reveal the directions of maximum diffusion. To best of our knowledge these components have never been utilized and experiments reveal they can be used for tracking white matter fibers and classification of tissues based upon the heterogeneity of water diffusion at voxel level. We are looking forward to see how the eigenvectors of these components can be used in tracking the fibers in heterogeneous regions.

\section{DISCLOSURES}

No conflicts of interest, financial or otherwise, are declared by the authors.

\section{ACKNOWLEDGMENTS}

The first two authors have been supported by the university grant MUNI/A/0885/2019 of Masaryk University, Jan Slovak gratefully acknowledges support from the Grant Agency of the Czech Republic, grant Nr.GA20-11473S. Sumit Kaushik acknowledges the support of the OP VVV funded project CZ.02.1.01/0.0/0.0/16-019/0000765 Research Center for for Informatics, Czech Technical University, Prague. The authors would like to thank Lubomir Vojtisek for providing real diffusion-weighted MRI data and acknowledge the core facility MAFIL of CEITEC supported by the MEYS CR (LM2018129 Czech-Bioimaging).

\section{REFERENCES}

[1] Vincent Arsigny, Pierre Fillard, Xavier Pennec, and Nicholas Ayache. 2006. LogEuclidean metrics for fast and simple calculus on diffusion tensors. Magnetic Resonance in Medicine: An Official Fournal of the International Society for Magnetic Resonance in Medicine 56, 2 (2006), 411-421.

[2] Angelos Barmpoutis, Min Sig Hwang, Dena Howland, John R Forder, and Baba C Vemuri. 2009. Regularized positive-definite fourth order tensor field estimation from DW-MRI. NeuroImage 45, 1 (2009), S153-S162.

[3] Angelos Barmpoutis and Baba C Vemuri. 2010. A unified framework for estimating diffusion tensors of any order with symmetric positive-definite constraints. In 2010 IEEE international symposium on biomedical imaging: from nano to macro. IEEE, 1385-1388.

[4] Bing Jian Barmpoutis and Baba C. Vemuri. 2009. Adaptive Kernels for Multi-fiber Reconstruction. Information processing in medical imaging : proceedings of the conference 21 (2009), 338-49.

[5] Peter J Basser, James Mattiello, and Denis LeBihan. 1994. Estimation of the effective self-diffusion tensor from the NMR spin echo. Journal of Magnetic Resonance, Series B 103, 3 (1994), 247-254.

[6] Peter J Basser and Sinisa Pajevic. 2003. A normal distribution for tensor-valued random variables: applications to diffusion tensor MRI. IEEE Transactions on Medical Imaging 22, 7 (2003), 785-794.
[7] George Keith Batchelor and I Proudman. 1956. The large-scale structure of homogenous turbulence. Philosophical Transactions of the Royal Society of London. Series A, Mathematical and Physical Sciences 248, 949 (1956), 369-405.

[8] Andrej Bóna, Ioan Bucataru, and Michael A Slawinski. 2004. Characterization of elasticity-tensor symmetries using SU (2). Journal of Elasticity 75, 3 (2004), 267-289.

[9] H Ertan Cetingül and René Vidal. 2011. Sparse Riemannian manifold clustering for HARDI segmentation. In 2011 IEEE International Symposium on Biomedical Imaging: From Nano to Macro. IEEE, 1750-1753.

[10] H Ertan Çetingül, Margaret J Wright, Paul M Thompson, and René Vidal. 2013. Segmentation of high angular resolution diffusion MRI using sparse Riemannian manifold clustering. IEEE transactions on medical imaging 33, 2 (2013), 301-317.

[11] Anne Collard, Silvere Bonnabel, Christophe Phillips, and Rodolphe Sepulchre. 2012. An anisotropy preserving metric for DTI processing. arXiv preprint arXiv:1210.2826 (2012).

[12] Stephen C Cowin. 1989. Properties of the anisotropic elasticity tensor. The Quarterly fournal of Mechanics and Applied Mathematics 42, 2 (1989), 249-266.

[13] P Thomas Fletcher. 2013. Geodesic regression and the theory of least squares on Riemannian manifolds. International journal of computer vision 105, 2 (2013), 171-185.

[14] P Thomas Fletcher and Sarang Joshi. 2004. Principal geodesic analysis on symmetric spaces: Statistics of diffusion tensors. In Computer Vision and Mathematical Methods in Medical and Biomedical Image Analysis. Springer, 87-98.

[15] P Thomas Fletcher and Sarang Joshi. 2007. Riemannian geometry for the statistical analysis of diffusion tensor data. Signal Processing 87, 2 (2007), 250-262.

[16] P Thomas Fletcher, Conglin Lu, and Sarang Joshi. 2003. Statistics of shape via principal geodesic analysis on Lie groups. In 2003 IEEE Computer Society Conference on Computer Vision and Pattern Recognition, 2003. Proceedings., Vol. 1. IEEE, I-I.

[17] Peter J, James Mattiello, and Denis LeBihan. 1994. MR diffusion tensor spectroscopy and imaging. Biophysical journal 66, 1 (1994), 259-267.

[18] L. Jonasson, X. Bresson, J. Thiran, V. J. Wedeen, and P. Hagmann. 2007. Representing Diffusion MRI in 5-D Simplifies Regularization and Segmentation of White Matter Tracts. IEEE Transactions on Medical Imaging 26, 11 (2007), 1547-1554.

[19] Sumit KAUSHIK. 2020 [cit. 2020-10-08]. Geometric Approach to Segmentation in Diffusion Magnetic Resonance Imaging [online]. Doctoral theses. Masaryk University, Faculty of Science, Brno. AvailablefromWWW<https://is.muni.cz/ th/thsvd/>

[20] Sumit Kaushik and Jan Slovák. 2019. HARDI Segmentation via Fourth-Order Tensors and Anisotropy Preserving Similarity Measures. Fournal of Mathematical Imaging and Vision 61 (2019), 1221 - 1234.

[21] Waseem Khan. 2013. Image segmentation techniques: A survey. Fournal of Image and Graphics 1, 4 (2013), 166-170.

[22] Kai Krajsek, Marion I Menzel, and Hanno Scharr. 2016. A Riemannian Bayesian framework for estimating diffusion tensor images. International fournal of Computer Vision 120, 3 (2016), 272-299.

[23] Paul C Lauterbur et al. 1973. Image formation by induced local interactions: examples employing nuclear magnetic resonance. (1973).

[24] AJBSJJDK Leemans, B Jeurissen, J Sijbers, and DK Jones. 2009. ExploreDTI: a graphical toolbox for processing, analyzing, and visualizing diffusion MR data. In Proc Intl Soc Mag Reson Med, Vol. 17. 3537.

[25] Christophe Lenglet, Mikaël Rousson, Rachid Deriche, Olivier Faugeras, Stéphane Lehericy, and Kamil Ugurbil. 2005. A Riemannian approach to diffusion tensor images segmentation. In Biennial International Conference on Information Processing in Medical Imaging. Springer, 591-602.

[26] Chunlei Liu, Roland Bammer, and Michael E Moseley. 2005. Limitations of apparent diffusion coefficient-based models in characterizing non-gaussian diffusion. Magnetic Resonance in Medicine: An Official fournal of the International Society for Magnetic Resonance in Medicine 54, 2 (2005), 419-428. 
[27] Morteza M Mehrabadi and Stephen C Cowin. 1990. Eigentensors of linear anisotropic elastic materials. The Quarterly fournal of Mechanics and Applied Mathematics 43, 1 (1990), 15-41.

[28] Maher Moakher. 2008. Fourth-order cartesian tensors: old and new facts, notions and applications. The Quarterly fournal of Mechanics \& Applied Mathematics 61, 2 (2008), 181-203.

[29] K. Ono, Yutaro Iwamoto, and Yen-Wei Chen. 2020. Automatic Segmentation of Infant Brain Ventricles with Hydrocephalus in MRI Based on 2.5D U-Net and Transfer Learning.

[30] Evren Özarslan and Thomas H Mareci. 2003. Generalized diffusion tensor imaging and analytical relationships between diffusion tensor imaging and high angular resolution diffusion imaging. Magnetic Resonance in Medicine: An Official fournal of the International Society for Magnetic Resonance in Medicine 50, 5 (2003), 955965.

[31] Sinisa Pajevic and Peter J Basser. 2003. Parametric and non-parametric statistical analysis of DT-MRI data. fournal of magnetic resonance 161, 1 (2003), 1-14.

[32] Xavier Pennec, Pierre Fillard, and Nicholas Ayache. 2006. A Riemannian framework for tensor computing. International fournal of computer vision 66, 1 (2006) 41-66.

[33] Lene Theil Skovgaard. 1984. A Riemannian geometry of the multivariate normal model. Scandinavian fournal of Statistics (1984), 211-223.
[34] Albert Tarantola. 2005. Elements for physics: quantities, qualities, and intrinsic theories. Springer Science \& Business Media.

[35] David Tschumperle and Rachid Deriche. 2001. Diffusion tensor regularization with constraints preservation. In Proceedings of the 2001 IEEE Computer Society Conference on Computer Vision and Pattern Recognition. CVPR 2001, Vol. 1. IEEE, I-I.

[36] David Solomon Tuch et al. 2002. Diffusion MRI of complex tissue structure. Ph.D. Dissertation. Massachusetts Institute of Technology.

[37] LJ Walpole. 1984. Fourth-rank tensors of the thirty-two crystal classes: multiplication tables. Proceedings of the Royal Society of London. A. Mathematical and Physical Sciences 391, 1800 (1984), 149-179.

[38] Van J Wedeen, Patric Hagmann, Wen-Yih Isaac Tseng, Timothy G Reese, and Robert M Weisskoff. 2005. Mapping complex tissue architecture with diffusion spectrum magnetic resonance imaging. Magnetic resonance in medicine 54, 6 (2005), 1377-1386.

[39] Yonas T Weldeselassie, Angelos Barmpoutis, and M Stella Atkins. 2012. Symmetric positive semi-definite cartesian tensor fiber orientation distributions (CT-FOD). Medical Image Analysis 16, 6 (2012), 1121-1129.

[40] Huatao Wu and Jonathan M Lees. 1999. Cartesian parametrization of anisotropic traveltime tomography. Geophysical fournal International 137, 1 (1999), 64-80. 